\title{
Changes in Electrical Activity of the Masseter Muscle and Masticatory Force after the Use of the Masseter Nerve as Donor in Facial Reanimation Surgery
}

\author{
Jose E. Telich-Tarriba' Alejandro Orihuela-Rodríguez ${ }^{2}$ \\ Fernando Ángeles-Medina ${ }^{3}$ Julio Morales-González ${ }^{3}$ \\ Damian Palafox ${ }^{1} \quad$ Alexander Cárdenas-Mejía ${ }^{1}$

\footnotetext{
${ }^{1}$ Division of Plastic and Reconstructive Surgery, Postgraduate Division of the Medical School, Hospital General "Dr Manuel Gea Gonzalez," Universidad Nacional Autonoma de Mexico,

Mexico City, Mexico

${ }^{2}$ Division of Stomatology and Orthodontics, Postgraduate Division of the School of Odontology, Hospital General "Dr Manuel Gea Gonzalez," Universidad Nacional Autonoma de Mexico,

Mexico City, Mexico

${ }^{3}$ Physiology Laboratory, Postgraduate Division of the School of Odontology, Hospital General "Dr Manuel Gea Gonzalez," Universidad Nacional Autonoma de Mexico, Mexico City, Mexico

${ }^{4}$ Department of Audiology, Instituto Nacional de Pediatria,

Mexico City, Mexico
}

Indian J Plast Surg 2020;53:59-63
Adriana de Lourdes Rivera-Priego ${ }^{2}$

Ignacio Mora-Magaña ${ }^{4}$ Adriana Fentanes-Vera ${ }^{1}$

\author{
Address for correspondence Alexander Cárdenas-Mejía, MD, Division of \\ Plastic and Reconstructive Surgery, Postgraduate Division of the Medical \\ School, Hospital General “Dr. Manuel Gea Gonzalez,” Universidad \\ Nacional Autonoma de Mexico, Calzada de Tlalpan 4800, 14080 \\ Mexico City, Mexico (e-mail: alexandercardenas@hotmail.com).
}

\section{Abstract}

Keywords

- masseter muscle

- bite force

- facial paralysis

- nerve transfer

- electromyography

- Bell's palsy

- masseter nerve

- neurorrhaphy

- gracilis muscle

- free functional muscle transfer
Introduction The masseter nerve has been used as a donor nerve for facial reanimation procedures due to the multiple advantages it offers; it has been generally considered that sacrifice of the masseter nerve does not alter the masticatory apparatus; however, there are no objective studies to support this claim.

Objective To evaluate the impact that the use of the masseter nerve in dynamic facial reconstruction has on the electrical activity of the masseter muscle and on bite force.

Materials and Methods An observational and prospective longitudinal study was performed measuring bite force and electrical activity of the masseter muscles before and 3 months after dynamic facial reconstructive surgery using the masseter nerve. An occlusal analyzer and surface electromyography were employed for measurements. Results The study included 15 patients with unilateral facial paralysis, with a mean age of $24.06 \pm 23.43$. Seven patients were subjected to a masseter-buccal branch nerve transfer, whereas in eight patients, the masseter nerve was used as a donor nerve for gracilis free functional muscle transfer. Electrical activity of the masseter muscle was significantly reduced after surgery in both occlusal positions: from $140.86 \pm 65.94$ to $109.68 \pm 68.04(p=0.01)$ in maximum intercuspation and from $123.68 \pm 75.64$ to $82.64 \pm 66.56(p=0.01)$ in the rest position. However, bite force did not show any reduction, changing from $22.07 \pm 15.66$ to $15.56 \pm 7.91(p=0.1)$ after the procedure.

Conclusion Masseter nerve transfer causes a reduction in electromyographic signals of the masseter muscle; however, bite force is preserved and comparable to preoperative status.
(C)2020 Association of Plastic Surgeons of India
License terms

() (1) $\Theta \circledast$ 


\section{Introduction}

Facial paralysis is a deeply disabling condition; adequate function of the mimetic muscles is essential for both verbal and nonverbal communication. Furthermore, the facial nerve is also responsible for providing the facial tone and movement necessary for ocular protection, nasal airflow, articulation of speech, and oral continence. ${ }^{1,2}$

Surgical management of facial paralysis sequelae remains challenging, and a wide variety of procedures for facial reanimation have been described; nevertheless, its main goals remain similar, such as restoration of facial symmetry at rest and the achievement of spontaneous and symmetric facial movements, with the smile being a prerogative., ${ }^{3,4}$

The use of extrafacial nerve donors in dynamic facial reanimation is indicated when the main trunk of the facial nerve is damaged or unavailable and/or the hypoglossal, contralateral facial, and spinal and masseter nerves have all been utilized with varying degrees of success; each nerve has inherent advantages and disadvantages. ${ }^{5}$

The masseter nerve has become a standard source of innervation in patients who are not candidates for cross-facial nerve grafts. The first description of its use in facial reanimation was made in 1925 by Escat and Viela; however, the procedure remained largely forgotten until Spira's preliminary reports in $1978 .^{6-8}$ The masseter nerve has several advantages that make it well suited for facial reanimation surgery, such as proximity to the facial nerve, consistent location, limited donor-site morbidity, high-density axonal load, and subsequent ease for rehabilitation..$^{9,10}$

One of the major disadvantages of the masseter nerve transfer is the loss of masseter muscle function; however, few authors have described if denervation of the masseter muscle has any functional impact on the rest of the masticatory apparatus. ${ }^{11}$ Masticatory function is the capacity of the individual to piece food; several factors such as age, number of teeth, and the physiology of saliva are associated with it. Bite force depends largely on the muscular elements that allow the temporomandibular joint to work appropriately, with the masseter being the most prominent muscle in this group. ${ }^{12}$ Is it possible that masseter muscle denervation alters the biomechanics of mastication, resulting in a reduction in bite force that could limit a patient's oral intake?

Literature related to changes in masseter muscle volume or bite force after masseter nerve harvesting are limited. Klebuc, and Hontanilla and Marre reported that patients in their clinical series rarely complained of masticatory problems., ${ }^{9,13}$ Yoshioka found that masseter nerve transfer causes masseter muscle atrophy in humans, ${ }^{14}$ whereas Carter and Harkness reported similar findings in a rat model. ${ }^{15}$ Despite these findings, no authors have reported if these morphological changes translate into functional alterations.

The "Dr Manuel Gea Gonzalez" General Hospital is a tertiary medical facility that provides medical attention mostly to the low-income uninsured population from the southern and eastern areas of Mexico City, and it is recognized as a national and international referral center for patients afflicted with facial paralysis. ${ }^{16}$ The objective of this study is to evaluate the impact that the use of the masseter nerve in dynamic facial reconstruction has on the electrical activity of the masseter muscle and on bite force.

\section{Methods}

An observational, prospective, longitudinal study was performed to evaluate the electrical activity of the masseter muscle and the mean bite force (MBF) in patients with facial paralysis planned for facial reanimation surgery using the masseter as a donor nerve at the Division of Plastic and Reconstructive Surgery in the "Dr. Manuel Gea Gonzalez" General Hospital in Mexico City during a 1-year period between January and December 2016.

Every patient with unilateral facial paralysis who was a candidate for dynamic facial reconstruction using the masseter as a donor nerve was included. Full approval by the institutional ethics board was received; consent was obtained from all patients or their guardians prior to inclusion in the study. Information was recorded using a data sheet including the following information: patient's age, gender, and side of paralysis; electrical activity (expressed as root mean square [RMS]); and bite force (expressed in kilogram-force $[\mathrm{kgF}])$. All measurements were obtained preoperatively and at 3 months after the surgical procedure was performed.

\section{Evaluation Techniques}

Electrical activity of the masseter muscles of both sides was measured by two-channel surface electromyography (EMG) with 20 - to $500-\mathrm{Hz}$ bandwidth, 100,000 amplification level, $127-V A C$ power supply, 12 bits, $2.44-\mathrm{mV}$ resolution, and $1,012 \Omega$ input impedance. Two Medi-Trace 100 Kendall MR (Covidien, Minneapolis, Minnesota, United States) surface electrodes were used over the masseter muscle, and a third electrode was placed on the surface of the ipsilateral mastoid process. Surface EMG was performed after asking the patient to bite at the maximum strength for 30 seconds first in the maximum intercuspation (MIC) and then in the rest position (RP); data were analyzed by calculating the RMS of the resulting values.

MBF was measured (in $\mathrm{kgF}$ ) using a computerized occlusal analyzer with T-Scan III sensor (Tekscan Inc., Boston, Massachusetts, United States), a device with 14,00 individual sensors and a resolution of $1.6 \mathrm{~mm}^{2}$ capable of measuring and generating global and individual maps of the distribution of masticatory force for the right and left sides. The tests were performed with the patient sitting with Frankfort's plane parallel to the ground; the sensor was introduced to the mouth and the patient as instructed to perform a strong bite for 5 seconds. Simultaneous measurements were performed on the right and left sides. ${ }^{17}$

\section{Surgical Technique}

Masseter nerve harvest is performed following Borschel and Zuker's technique. A preauricular incision is performed and the fascia is spread parallel to the zygomatic arch at a point $3 \mathrm{~cm}$ anterior to the tragus and $1 \mathrm{~cm}$ inferior to the zygomatic arch. The masseter muscle is bluntly dissected 
until the nerve is located. Once the nerve is identified, it is dissected free of muscle until it begins to divide into small branches and is then transected and mobilized to perform the neurorrhaphy. ${ }^{18}$

\section{Statistical Analysis}

A database was created on Microsoft Office Access 2007, and data were analyzed on the MATLAB software, version 2011, for 32-bit systems (MathWorks Inc., Natick, Massachusetts, United States). Descriptive analyses of patient demographic and clinical characteristics were performed. Continuous variables are expressed in measures of central tendency, and categorical values are presented as percentages. Comparative statistics between pre- and postoperative values were performed using Wilcoxon's signed-rank test since our sample presented a nonparametric distribution, as determined by the Shapiro-Wilk test. A $p$-value of $<0.05$ was considered significant.

\section{Results}

A total of 15 patients ( 9 females and 6 males) were included in this study, with an average age of $24.06 \pm 23.43$ years. In nine cases, the paralysis was left-sided, whereas in the rest of the patients, it was right-sided. Etiology of the paralysis was developmental in nine patients, Bell's palsy in five patients, and in one individual it developed after a mastoidectomy. Seven patients were subjected to a masseter-buccal branch nerve transfer, and in eight cases, the masseter nerve was used as a donor nerve for gracilis muscle transfer ( - Table $\mathbf{1}$ ).

Preoperative surface EMG at MIC was $140.86 \pm 65.94$ on the side with paralysis and $141.7 \pm 71.3$ on the healthy side; after surgery, the value on the paralyzed significantly decrease to $109.68 \pm 68.04(p=0.01)$, whereas the contralateral side did not show significant variation $(150.44 \pm 54.9 ; p=0.3$; - Fig. 1 ).
EMG results during RP were $123.68 \pm 75.64$ for the affected side and $122.59 \pm 74.55$ for the healthy one. Postoperative values for the operated side significantly decreased to $82.64 \pm 66.56(p=0.01)$, no significant changes were observed on the nonoperated side (136.75 $\pm 65.88 ; p=0.2 ;$ - Fig. 2 ).

In light of these data, it was decided to perform a posthoc analysis comparing the postoperative results of both sides of the face, which revealed that EMG values on the intervened side were significantly lower in both MIC $(p=0.01)$ and RP $(p=0.02)$.

Preoperative MBF was $22.67 \pm 16.69$ on the paralyzed side and $22.07 \pm 15.66$ on the nonparalyzed side; postoperative results showed a decrease in MBF on both the paralyzed (15.56 \pm 7.91$)$ and healthy sides (15.56 \pm 6.88$)$; however, no statistical significance was found after comparing pre- and postoperative values ( $p=0.1$ ) nor between the normal and the intervened side ( $p=0.9 ;-$ Fig. 3 ).

\section{Discussion}

Facial paralysis is a devastating disease and presents a formidable challenge in terms of treatment. A wide variety of surgical options for facial reanimation have been developed; historically, the hypoglossal or accessory nerves were used as extrafacial nerve donors, but in recent years, interest in the masseter nerve has grown due to its several advantages such as proximity to the facial nerve, constant anatomical location, ease of mobilizations that allows direct anastomosis to the buccal branch, and possessing an axonal load of more than 2,700 fibers. ${ }^{18,19}$

The main proponents of the technique indicate that surgical site morbidity is minimal after masseter nerve transfer, arguing that any changes in the electrical activity and strength of the masseter are negligible and can be compensated by the redundant function of the temporalis

Table 1 Clinical characteristics of the patients included in the study

\begin{tabular}{|l|l|l|l|l|l|}
\hline Patient & Gender & Age & Etiology & Paralyzed side & Procedure \\
\hline 1 & M & 5 & Developmental & R & FFMT \\
\hline 2 & F & 46 & Postoperative & L & NT \\
\hline 3 & F & 11 & Developmental & R & NT \\
\hline 4 & F & 14 & Developmental & L & FFMT \\
\hline 5 & F & 35 & Bell's palsy & R & NT \\
\hline 6 & F & 6 & Developmental & L & FFMT \\
\hline 7 & F & 67 & Bell's palsy & L & NT \\
\hline 8 & M & 7 & Developmental & L & FFMT \\
\hline 9 & F & 56 & Bell's palsy & R & NT \\
\hline 10 & M & 10 & Developmental & L & FFMT \\
\hline 11 & F & 15 & Developmental & L & NT \\
\hline 12 & M & 9 & Bell's palsy & L & FFMT \\
\hline 13 & F & 5 & Developmental & L & NT \\
\hline 14 & M & 8 & Developmental & R & FFMT \\
\hline 15 & M & 67 & Bell's palsy & R & FFMT \\
\hline
\end{tabular}

Abbreviations: FFMT, free functional muscle transfer; L, Left; NT, nerve transfer; R, right. 




Fig. 1 Results of surface electromyography of the masseter muscle during maximal intercuspation, showing a significant decrease in the electrical activity of the paralyzed side after the surgical procedure. EMG, electromyography; RMS, root mean square.

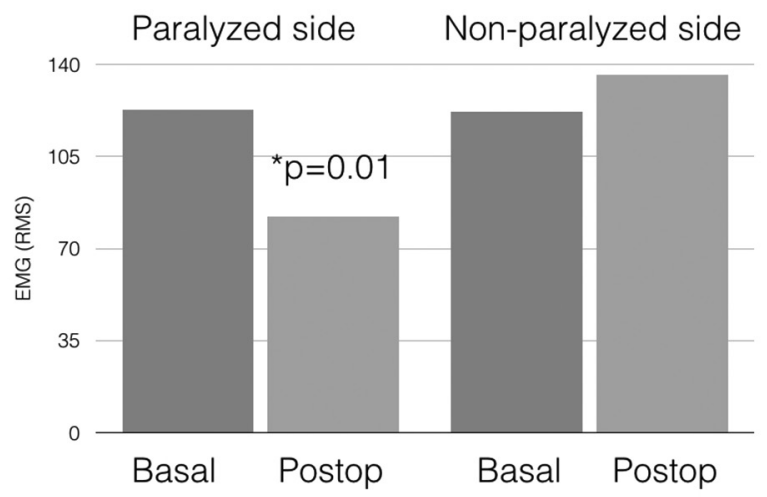

Fig. 2 Results of surface electromyography of the masseter muscle during the rest position, showing a significant decrease in the electrical activity of the paralyzed side after the surgical procedure. EMG, electromyography; RMS, root mean square.

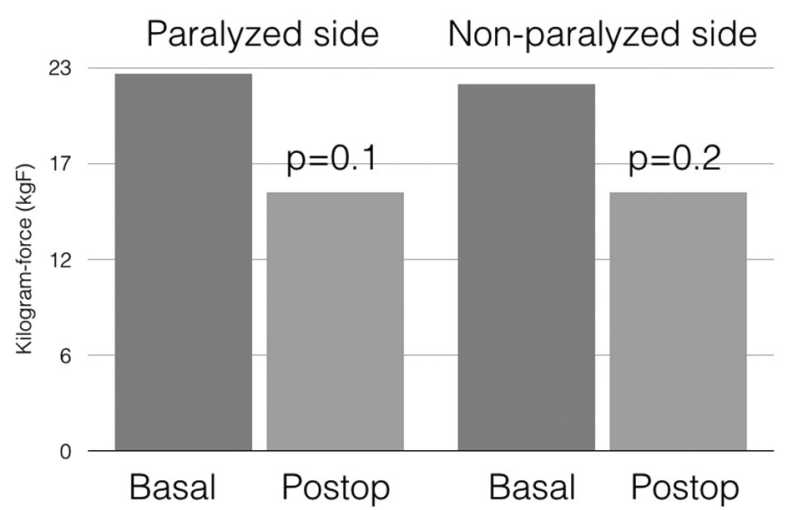

Fig. 3 Bite force results before and after surgery, showing no significant alterations on the paralyzed side.

and pterygoid muscles ${ }^{18}$; nevertheless, objective evidence in regard to the functional changes caused by masseter denervation is lacking.

Most studies have focused solely on subjective reports by patients or on evaluation of the muscle's morphology; for example, Klebuc reported in a 12-year review of clinical experience that no difficulties with temporomandibular joint function or mastication had been found, ${ }^{9}$ whereas Hontanilla and Cabello noted that patients seldom complained of masticatory force reduction after the procedure. ${ }^{10}$ A study by Carter et al in rats found that masseter muscle denervation causes masseter muscle atrophy and compensatory hypertrophy of the temporalis ${ }^{15}$; these findings were later corroborated in humans by Yoshioka, who evaluated changes in muscle area after masseteric nerve transfer using computed tomography (CT) scans. ${ }^{14}$

Development of the evaluation techniques for this study was previously applied and standardized by the research team. ${ }^{12,17}$ Surface EMG has been widely used for the evaluation of the masseter muscle in international literature and proven to be reliable due to the volume and superficial position of the muscle. Since bite force assessment can be altered due to several variables such as pain, dental status, and periodontal support, each patient served as a self-control, thus eliminating any interpersonal differences..$^{20,21}$

Our study showed that the electrical activity of the masseter muscle is globally diminished after harvesting the masseteric nerve. Reduction of electromyographic activity clearly develops due to denervation of the muscle, and whether it is partial or total, it depends on each patient's individual anatomy. Brenner and Schoeller found that the masseter nerve has two or more branches in $75 \%$ of the cases, ${ }^{8}$ whereas Borschel et al stated that the first extracranial branch of the motor nerve to the masseter muscle arises deep to the zygomatic arch and is not disrupted during classical masseter nerve harvesting. ${ }^{18}$

Despite the reduction in the electrical activity of the masseter muscle, this did not translate into an overall loss of bite force, and several mechanisms could be responsible for this finding. As previously stated, it is possible that the denervation of the masseter muscle is only partial, thus maintaining its strength. ${ }^{18}$ It is also possible that the remaining masticatory muscles hypertrophy and compensate for the loss of strength of the masseter muscle, as supported by morphological evidence from Yoshioka, and Carter and Harkness. ${ }^{14,15}$

Our study's main limitation is the small sample size; nonetheless, it should be noted that extrafacial donor nerves are used when the facial nerve is not available, and therefore the use of the masseter nerve accounts for a fraction of all dynamic facial reconstruction performed at our institution.

We consider that our study presents several strengths such as its prospective design and, most importantly, the fact that it is the first study that evaluates the functional impact of using the masseter as a donor nerve in an objective manner. Further research in our group is focused on identifying the effects that masseter nerve transfer might have on long-term skeletal development in pediatric patients.

\section{Conclusion}

Masseter nerve transfer causes a reduction in electromyographic signals of the masseter muscle; however, masticatory force is preserved and comparable to preoperative status. 


\section{Note}

This paper was presented at the American Society for Peripheral Nerve's Annual Meeting, Phoenix, Arizona, United States, January 12 to 14, 2018.

\section{Ethical Statement}

Full approval by the institutional ethics board was received; consent was obtained from all patients or their guardians prior to inclusion in the study.

\section{Funding}

No funding was received for this article.

\section{Conflict of Interest}

The authors have no conflicts of interest to disclose.

\section{References}

1 Fattah A, Borschel GH, Manktelow RT, Bezuhly M, Zuker RM. Facial palsy and reconstruction. Plast Reconstr Surg 2012;129(2):340e-352e

2 Coombs CJ, Ek EW, Wu T, Cleland H, Leung MK. Masseteric-facial nerve coaptation-an alternative technique for facial nerve reinnervation. J Plast Reconstr Aesthet Surg 2009;62(12):1580-1588

3 Terzis JK, Noah ME. Analysis of 100 cases of free-muscle transplantation for facial paralysis. Plast Reconstr Surg 1997;99(7):1905-1921

4 Biglioli F, Frigerio A, Rabbiosi D, Brusati R. Single-stage facial reanimation in the surgical treatment of unilateral established facial paralysis. Plast Reconstr Surg 2009;124(1):124-133

5 Klebuc M, Shenaq SM. Donor nerve selection in facial reanimation surgery. Semin Plast Surg 2004;18(1):53-60

6 Yoshioka N, Tominaga S. Masseteric nerve transfer for short-term facial paralysis following skull base surgery. J Plast Reconstr Aesthet Surg 2015;68(6):764-770

7 Biglioli F, Colombo V, Tarabbia F, et al. Recovery of emotional smiling function in free-flap facial reanimation. J Oral Maxillofac Surg 2012;70(10):2413-2418

8 Brenner E, Schoeller T. Masseteric nerve: a possible donor for facial nerve anastomosis? Clin Anat 1998;11(6):396-400

9 Klebuc M. The evolving role of the masseter-to-facial (V-VII) nerve transfer for rehabilitation of the paralyzed face. Ann Chir Plast Esthet 2015;60(5):436-441
10 Hontanilla B, Cabello A. Spontaneity of smile after facial paralysis rehabilitation when using a non-facial donor nerve. J Craniomaxillofac Surg 2016;44(9):1305-1309

11 Rich ME. Masseter muscle bite force in first bicuspid and collapsed occlusion cases. Int $\mathrm{J}$ Orthod Milwaukee 2012;23(2):29-33

12 Telich-Tarriba JE, Contreras-Molinar C, Orihuela-Rodriguez A. Lesta-Compagnucci L, Carrillo-Cordova JR, Cardenas-Mejia A. Bite force and electromyographic activity of the masseter muscle in children with hemifacial microsomia. J Plast Surg Hand Surg 2019;53(5):316-319

13 Hontanilla B, Marre D. Masseteric-facial nerve transposition for reanimation of the smile in incomplete facial paralysis. Br J Oral Maxillofac Surg 2015;53(10):943-948

14 Yoshioka N. Masseter atrophication after masseteric nerve transfer. Is it negligible? Plast Reconstr Surg Glob Open 2016;4(4):e692

15 Carter GM, Harkness EM. Alterations to mandibular form following motor denervation of the masseter muscle. An experimental study in the rat. J Anat 1995;186(Pt 3):541-548

16 Telich-Tarriba JE, Velazquez E, Theurel-Cuevas A, et al. Upper extremity patterns of injury and management at a plastic and reconstructive surgery referral center in Mexico City. Ann Plast Surg 2018;80(1):23-26

17 Ortega -Robles ED. Diseño y construcción de un sistema computarizado para el análisis de las fuerzas generadas entre los dientes superiores e inferiores durante la oclusión [Thesis]. Centro de Investigacion y Estudios Avanzados del Instituto Politecnico Nacional Mexico City, Mexico; 2012

18 Borschel GH, Kawamura DH, Kasukurthi R, Hunter DA, Zuker RM, Woo AS. The motor nerve to the masseter muscle: an anatomic and histomorphometric study to facilitate its use in facial reanimation. J Plast Reconstr Aesthet Surg 2012;65(3):363-366

19 Murphey AW, Clinkscales WB, Oyer SL. Masseteric nerve transfer for facial nerve paralysis: a systematic review and meta-analysis. JAMA Facial Plast Surg 2018;20(2):104-110

20 Koc D, Dogan A, Bek B. Bite force and influential factors on bite force measurements: a literature review. Eur J Dent 2010;4(2):223-232

21 Jian C, Wei M, Luo J, et al. Multiparameter electromyography analysis of the masticatory muscle activities in patients with brainstem stroke at different head positions. Front Neurol 2017;8:221 